\title{
Quadratic-Argument Approach to Nonlinear Schrödinger Equation and Coupled Onesı
}

\author{
Xiaoping Xu \\ Institute of Mathematics, Academy of Mathematics \& System Sciences \\ Chinese Academy of Sciences, Beijing 100190, P.R. China 2
}

\begin{abstract}
The two-dimensional cubic nonlinear Schrödinger equation is used to describe the propagation of an intense laser beam through a medium with Kerr nonlinearity. The coupled twodimensional cubic nonlinear Schrödinger equations are used to describe interaction of electromagnetic waves with different polarizations in nonlinear optics. In this paper, we solve the above equations by imposing a quadratic condition on the related argument functions and using their symmetry transformations. More complete families of exact solutions of such type are obtained. Many known interesting solutions, such soliton ones, turn out to be special cases of our solutions.
\end{abstract}

\section{Introduction}

The two-dimensional cubic nonlinear Schrödinger equation:

$$
i \psi_{t}+c\left(\psi_{x x}+\psi_{y y}\right)+a|\psi|^{2} \psi=0
$$

is used to describe the propagation of an intense laser beam through a medium with Kerr nonlinearity, where $t$ is the distance in the direction of propagation, $x$ and $y$ are the transverse spacial coordinates, $\psi$ is a complex valued function in $t, x, y$ standing for electric field amplitude, and $a, c$ are nonzero real constants. We refer the introduction of [SEG] for more systematic exposition of the equation. Akhnediev, Eleonskii and Kulagin [AEK] found certain exact solutions of (1.1) whose real and imaginary parts are linearly dependent over the functions of $t$. Moreover, Gagnon and Winternitz [GW] found exact

\footnotetext{
${ }^{1} 2000$ Mathematical Subject Classification. Primary 35C05, 35Q55; Secondary 37K10.

${ }^{2}$ Research supported by China NSF 10431040
} 
solutions of the cubic and quintic nonlinear Schrödinger equation for a cylindrical geometry. Mihalache and Panoin [MN] used the method in [AEK] to obtain new solutions which describe the propagation of dark envelope soliton light pulses in optical fibers in the normal group velocity dispersion regime. Furthermore, Saied, EI-Rahman and Ghonamy [SEG] used various similarity variables to reduce the above equation to certain ordinary differential equations and obtain some exact solutions. However, many of their solutions are equivalent to each other under the action of the known symmetry transformations of the above equation.

The coupled two-dimensional cubic nonlinear Schrödinger equations

$$
\begin{aligned}
& i \psi_{t}+c_{1}\left(\psi_{x x}+\psi_{y y}\right)+\left(a_{1}|\psi|^{2}+b_{1}|\varphi|^{2}\right) \psi=0, \\
& i \varphi_{t}+c_{2}\left(\varphi_{x x}+\varphi_{y y}\right)+\left(a_{2}|\psi|^{2}+b_{2}|\varphi|^{2}\right) \varphi=0
\end{aligned}
$$

are used to describe interaction of electromagnetic waves with different polarizations in nonlinear optics, where $a_{1}, a_{2}, b_{1}, b_{2}, c_{1}$ and $c_{2}$ are real constants. Radhakrishnan and Lakshmanan [RL1] used Painlevé analysis to find a Hirota bilinearization of the above system of partial differential equations and obtained bright and dark multiple soliton soutions. They also generalized their results to the coupled nonlinear Schrödinger equations with higher-order effects in [RL2]. Grébert and Guillot [GG] construcetd periodic solutions of coupled one-dimensional nonlinear Schrödinger equations with periodic boundary conditions in some resonance situations. Moreover, Hioe and Salter [HS] found a connections between Lamé functions and solutions of the above coupled equations.

In terms of real-valued functions, the above equations form systems of nonlinear partial differential equations. Such systems can not be solved exactly without pre-assumptions. We observe that the argument functions of many known solutions for these equations are quadratic in the spacial variables $x$ and $y$, in particular, those in [SEG]. Moreover, some of these solutions are actually equivalent to each other under the Lie point symmetries of their corresponding equations. These facts motivate us to solve the above equations in this paper by imposing the quadratic condition on the related argument functions and using their symmetry transformations. More complete families of explicit exact solutions of this type with multiple parameter functions are obtained. Many known interesting solutions, such soliton ones, turn out to be special cases of our solutions. Various singular solutions and periodic solutions that we obtain may reflect some important physical phenomena in practical models. Our solutions can also be used to solve some boundary-value problems. Below we give more details. 
For convenience, we always assume that all the involved partial derivatives of related functions always exist and we can change orders of taking partial derivatives. We also use prime' to denote the derivative of any one-variable function.

It is known that the equation (1.1) is invariant under the following known symmetric transformations:

$$
\begin{gathered}
T_{1}(\psi)=d e^{d_{3} i} \psi\left(d^{2} t+d_{2}, d\left(x \cos d_{1}+y \sin d_{1}\right), d\left(-x \sin d_{1}+y \cos d_{1}\right)\right), \\
T_{2}(\psi)=e^{\left[2\left(d_{1} x+d_{3} y\right)-\left(d_{1}^{2}+d_{3}^{2}\right) t\right] i / 4 c} \psi\left(t, x-d_{1} t+d_{2}, y-d_{3} t+d_{4}\right),
\end{gathered}
$$

where $d, d_{1}, d_{2}, d_{3}, d_{4} \in \mathbb{R}$ with $d \neq 0$. In other words, the above transformations transform one solution of (1.1) into another solution. Our solutions contain all the solutions in [SEG] up to the above transformations. In particular, our solutions with elliptic functions were not given in [SEG]. Our approach is quite elementary and accessible to large audiences such as physicists and engineers. For the reader's convenience, we list in this paper all the solutions of the equation (1.1) found by our method although some of them are known and obvious. This may help non-mathematicians to apply the solutions of the Schrödinger equation to their fields. In fact, applying the transformations in (1.4) and (1.5) to any of our solutions will yield more sophisticated one.

Similarly, we have the following known symmetric transformations of the coupled equations (1.2) and (1.3):

$$
\begin{gathered}
T_{1}(\psi)=d e^{d_{3} i} \psi\left(d^{2} t+d_{2}, d\left(x \cos d_{1}+y \sin d_{1}\right), d\left(-x \sin d_{1}+y \cos d_{1}\right)\right), \\
T_{1}(\varphi)=d e^{d_{4} i} \varphi\left(d^{2} t+d_{2}, d\left(x \cos d_{1}+y \sin d_{1}\right), d\left(-x \sin d_{1}+y \cos d_{1}\right)\right) ; \\
T_{2}(\psi)=e^{\left[2\left(d_{1} x+d_{3} y\right)-\left(d_{1}^{2}+d_{3}^{2}\right) t\right] i / 4 c_{1}} \psi\left(t, x-d_{1} t+d_{2}, y-d_{3} t+d_{4}\right), \\
T_{2}(\varphi)=e^{\left[2\left(d_{1} x+d_{3} y\right)+\left(d_{1}^{2}+d_{3}^{2}\right) t\right] i / 4 c_{2}} \varphi\left(t, x-d_{1} t+d_{2}, y-d_{3} t+d_{4}\right) ;
\end{gathered}
$$

where $d, d_{1}, d_{2}, d_{3}, d_{4} \in \mathbb{R}$ with $d \neq 0$. In addition to the above symmetries, we also solve the coupled equations modulo the following symmetry:

$$
\left(\psi, a_{1}, b_{1}, c_{1}\right) \leftrightarrow\left(\varphi, a_{2}, b_{2}, c_{2}\right)
$$

Again for the reader's convenience, we list in this paper all the solutions of the coupled equations (1.2) and (1.3) found by our method although some of them are known and obvious. For convenience, we always assume that all the involved partial derivatives of related functions always exist and we can change orders of taking partial derivatives. We also use prime' to denote the derivative of any one-variable function.

In Section 2, we solve the Schrödinger equation (1.1). In Section 3, we use the results in Section 2 to solve the coupled Schrödinger equations (1.2) and (1.3). 


\section{Exact Solutions of the Schrödinger Equation}

In this section, we will present our quadratic-argument approach to the two-dimensional cubic nonlinear Schrödinger equation (1.1) and find more exact solutions than [SEG] in the modulo sense.

Write

$$
\psi=\xi(t, x, y) e^{i \phi(t, x, y)}
$$

where $\xi$ and $\phi$ are real functions in $t, x, y$. Note

$$
\begin{gathered}
\psi_{t}=\left(\xi_{t}+i \xi \phi_{t}\right) e^{i \phi}, \quad \psi_{x}=\left(\xi_{x}+i \xi \phi_{x}\right) e^{i \phi}, \quad \psi_{y}=\left(\xi_{y}+i \xi \phi_{y}\right) e^{i \phi} \\
\psi_{x x}=\left(\xi_{x x}-\xi \phi_{x}^{2}+i\left(2 \xi_{x} \phi_{x}+\xi \phi_{x x}\right)\right) e^{i \phi}, \quad \psi_{y y}=\left(\xi_{y y}-\xi \phi_{y}^{2}+i\left(2 \xi_{y} \phi_{y}+\xi \phi_{y y}\right)\right) e^{i \phi} .
\end{gathered}
$$

So the equation (1.1) becomes

$$
\begin{aligned}
& i \xi_{t}-\phi_{t} \xi+a \xi^{3}+c\left[\xi_{x x}+\xi_{y y}-\xi\left(\phi_{x}^{2}+\phi_{y}^{2}\right)\right. \\
& \left.+i\left(2 \xi_{x} \phi_{x}+2 \xi_{y} \phi_{y}+\xi\left(\phi_{x x}+\phi_{y y}\right)\right)\right]=0
\end{aligned}
$$

equivalently,

$$
\begin{gathered}
\xi_{t}+c\left(2 \xi_{x} \phi_{x}+2 \xi_{y} \phi_{y}+\xi\left(\phi_{x x}+\phi_{y y}\right)\right)=0, \\
-\xi\left[\phi_{t}+c\left(\phi_{x}^{2}+\phi_{y}^{2}\right)\right]+c\left(\xi_{x x}+\xi_{y y}\right)+a \xi^{3}=0 .
\end{gathered}
$$

Note that it is very difficult to solve the above system without pre-assumptions. We observe that the functions $\phi$ in all the solutions of [SEG] are quadratic in $x$ and $y$. From the algebraic characteristics of the above system of partial differential equations, it is most affective to assume that $\phi$ is quadratic in $x$ and $y$. After sorting case by case, we only have the following four cases that lead us to exact solutions, modulo the transformations in (1.4) and (1.5).

Case 1. $\phi=\beta(t)$ is a function of $t$.

According to $(2.5), \xi_{t}=0$. Moreover, (2.6) becomes

$$
-\beta^{\prime} \xi+c\left(\xi_{x x}+\xi_{y y}\right)+a \xi^{3}=0
$$

So we take

$$
\beta=b t+d, \quad b, d \in \mathbb{R} .
$$

If $b=0$ and $a c<0$, modulo the transformations (1.4), we take $d=0$ and the following solutions:

$$
\xi=\frac{1}{x} \sqrt{-\frac{2 c}{a}} \quad \text { or } \quad \sqrt{-\frac{c}{a\left(x^{2}+y^{2}\right)}} .
$$


Next we assume $b \neq 0$. Modulo the transformation (1.4), we can take $d=0$. Note that

$$
\begin{gathered}
(\tan s)^{\prime \prime}=2\left(\tan ^{3} s+\tan s\right), \quad(\sec s)^{\prime \prime}=2 \sec ^{3} s-\sec s \\
(\operatorname{coth} s)^{\prime \prime}=2\left(\operatorname{coth}^{3} s-\operatorname{coth} s\right), \quad(\operatorname{csch} s)^{\prime \prime}=2 \operatorname{csch}^{3} s+\operatorname{csch} s .
\end{gathered}
$$

Denote Jacobi elliptic functions

$$
\text { sn } s=\operatorname{sn}(s \mid m), \quad \operatorname{cn} s=\operatorname{cn}(s \mid m), \quad \operatorname{dn} s=\operatorname{dn}(s \mid m),
$$

where $m$ is the elliptic modulus (e.g., cf. [WG]). Then

$$
\begin{gathered}
(\operatorname{sn} s)^{\prime \prime}=2 m^{2} \operatorname{sn}^{3} s-\left(1+m^{2}\right) \operatorname{sn} s \\
(\mathrm{cn} s)^{\prime \prime}=-2 m^{2} \mathrm{cn}^{2} s+\left(2 m^{2}-1\right) \mathrm{cn} s \\
(\operatorname{dn} s)^{\prime \prime}=-2 \operatorname{dn}^{3} s+\left(2-m^{2}\right) \operatorname{dn} s .
\end{gathered}
$$

Moreover,

$$
\lim _{m \rightarrow 1} \operatorname{sn} s=\tanh s, \quad \lim _{m \rightarrow 1} \operatorname{cn} s=\lim _{m \rightarrow 1} \operatorname{dn} s=\operatorname{sech} s .
$$

Consider solutions modulo the transformation (1.4). If $a c<0$, we have the following solutions:

$$
\begin{gathered}
\xi=\sqrt{-\frac{2 c}{a}} \tan x, \quad b=2 c ; \\
\xi=\sqrt{-\frac{2 c}{a}} \sec x, \quad b=-c ; \\
\xi=\sqrt{-\frac{2 c}{a}} \operatorname{coth} x, \quad b=-2 c ; \\
\xi=\sqrt{-\frac{2 c}{a}} \operatorname{csch} x, \quad b=c ; \\
\xi=m \sqrt{-\frac{2 c}{a}} \operatorname{sn} x, \quad b=-\left(1+m^{2}\right) c .
\end{gathered}
$$

When $a c>0$, we get the following solutions:

$$
\begin{array}{cl}
\xi=m \sqrt{\frac{2 c}{a}} \operatorname{cn} x, & b=\left(2 m^{2}-1\right) c, \\
\xi & =\sqrt{\frac{2 c}{a}} \operatorname{dn} x, \quad b=\left(2-m^{2}\right) c .
\end{array}
$$

Theorem 2.1.Let $m \in \mathbb{R}$. The following function are solutions $\psi$ of the two-dimensional cubic nonlinear cubic nonlinear Schrödinger equation (1.1): if ac $<0$,

$$
\frac{1}{x} \sqrt{-\frac{2 c}{a}}, \quad \sqrt{-\frac{c}{a\left(x^{2}+y^{2}\right)}}, \quad e^{2 c t i} \sqrt{-\frac{2 c}{a}} \tan x, \quad e^{-c t i} \sqrt{-\frac{2 c}{a}} \sec x
$$




$$
e^{-2 c t i} \sqrt{-\frac{2 c}{a}} \operatorname{coth} x, \quad e^{c t i} \sqrt{-\frac{2 c}{a}} \operatorname{csch} x, \quad m e^{-\left(1+m^{2}\right) c t i} \sqrt{-\frac{2 c}{a}} \operatorname{sn} x
$$

when $a c>0$,

$$
m e^{\left(2 m^{2}-1\right) c t i} \sqrt{\frac{2 c}{a}} \operatorname{cn} x, \quad e^{\left(2-m^{2}\right) c t i} \sqrt{\frac{2 c}{a}} \operatorname{dn} x .
$$

Remark 2.2. Although the above solution are simple, we can obtain more sophisticated ones by applying the transformations (1.4) and (1.5) to them. For instance, applying the transformation (1.4) to the first solution in (2.24), we get a solution:

$$
\psi=\frac{e^{d_{2} i}}{x \cos d_{1}+y \sin d_{1}} \sqrt{-\frac{2 c}{a}}, \quad d_{1}, d_{2} \in \mathbb{R} .
$$

Applying the transformation (1.5) to the above solution, we obtain another solution:

$$
\psi=\frac{e^{\left[2\left(d_{3} x+d_{4} y\right)+\left(d_{3}^{2}+d_{4}^{2}\right) t+d_{2}\right] i / 4 c}}{\left(x-d_{3} t\right) \cos d_{1}+\left(y-d_{4} t\right) \sin d_{1}+d_{5}} \sqrt{-\frac{2 c}{a}}, \quad d_{1}, d_{2}, d_{3}, d_{4}, d_{5} \in \mathbb{R} .
$$

Case 2. $\phi=x^{2} / 4 c t+\beta$ for some function $\beta$ of $t$.

In this case, (2.5) becomes

$$
\xi_{t}+\frac{x}{t} \xi_{x}+\frac{1}{2 t} \xi=0
$$

Thus

$$
\xi=\frac{1}{\sqrt{t}} \zeta(u, y), \quad u=\frac{x}{t},
$$

for some two-variable function $\zeta$. Now (2.6) becomes (2.7). Note

$$
\xi_{x x}=t^{-5 t / 2} \zeta_{\text {uu }}, \quad \xi_{y y}=t^{-1 / 2} \zeta_{y y}, \quad \xi^{3}=t^{-3 / 2} \zeta^{3} .
$$

So (2.7) becomes

$$
-\frac{\beta^{\prime}}{\sqrt{t}} \zeta+c\left(t^{-5 t / 2} \zeta_{u u}+t^{-1 / 2} \zeta_{y y}\right)+a t^{-3 / 2} \zeta^{3}=0
$$

whose coefficients of $t^{-3 / 2}$ force us to take

$$
\xi=\frac{b}{\sqrt{t}}, \quad b \in \mathbb{R} .
$$

Now (2.7) becomes

$$
-\beta^{\prime}+\frac{a b^{2}}{t}=0 \Longrightarrow \beta=a b^{2} \ln t
$$


modulo the transformation in (1.5).

Case 3. $\phi=x^{2} / 4 c t+y^{2} / 4 c(t-d)+\beta$ for some function $\beta$ of $t$ with $0 \neq d \in \mathbb{R}$.

In this case, (2.5) becomes

$$
\xi_{t}+\frac{x}{t} \xi_{x}+\frac{y}{t-d} \xi_{y}+\left(\frac{1}{2 t}+\frac{1}{2(t-d)}\right) \xi=0 .
$$

So we have:

$$
\xi=\frac{1}{\sqrt{t(t-d)}} \zeta(u, v), \quad u=\frac{x}{t}, v=\frac{y}{t-d},
$$

for some two-variable function $\zeta$. Again (2.6) becomes (2.7). Note

$$
\xi_{x x}=t^{-5 / 2}(t-c)^{-1 / 2} \zeta_{u u}, \quad \xi_{y y}=t^{-1 / 2}(t-c)^{-5 / 2} \zeta_{v v}, \quad \xi^{3}=t^{-3 / 2}(t-c)^{-3 / 2} \zeta^{3} .
$$

So (2.7) becomes

$$
-\frac{\beta^{\prime}}{\sqrt{t(t-d)}} \zeta+c\left(t^{-5 / 2}(t-c)^{-1 / 2} \zeta_{u u}+t^{-1 / 2}(t-c)^{-5 / 2} \zeta_{v v}\right)+a t^{-3 / 2}(t-c)^{-3 / 2} \zeta^{3}=0
$$

whose coefficients of $t^{-3 / 2}(t-c)^{-3 / 2}$ force us to take

$$
\xi=\frac{b}{\sqrt{t(t-d)}}, \quad b \in \mathbb{R}
$$

Now (2.7) becomes

$$
-\beta^{\prime}+\frac{a b^{2}}{t(t-d)}=0 \Longrightarrow \beta=\frac{a b^{2}}{d} \ln \frac{t-d}{t}
$$

modulo the transformation in (1.5).

Theorem 2.3. Let $b, d \in \mathbb{R}$ with $d \neq 0$. The following function are solutions $\psi$ of the two-dimensional cubic nonlinear cubic nonlinear Schrödinger equation:

$$
b t^{a b^{2} i-1 / 2} e^{x^{2} i / 4 c t}, \quad b t^{-a b^{2} i / d-1 / 2}(t-d)^{a b^{2} i / d-1 / 2} e^{x^{2} i / 4 c t+y^{2} i / 4 c(t-d)} .
$$

Remark 2.4. Applying (1.4) to the above first solution, we get another solution

$$
\psi=d d_{1}\left(d_{1}^{2} t+d_{4}\right)^{a d^{2} i-1 / 2} \exp \left(\frac{d_{1}^{2}\left(x \cos d_{2}+y \sin d_{2}\right)^{2}}{4 c\left(d_{1}^{2} t+d_{4}\right)}+d_{3}\right) i,
$$

for $d_{1}, d_{2}, d_{3}, d_{4} \in \mathbb{R}$. Moreover, we obtain a more sophisticated solution:

$$
\begin{aligned}
\psi= & d d_{1}\left(d_{1}^{2} t+d_{4}\right)^{a d^{2} i-1 / 2} \exp \frac{d_{1}^{2}\left(\left(x-d_{5} t\right) \cos d_{2}+\left(y-d_{6} t\right) \sin d_{2}+d_{7}\right)^{2} i}{4 c\left(d_{1}^{2} t+d_{4}\right)} \\
& \times \exp \left(\frac{2\left(d_{5} x+d_{6} y\right)+\left(d_{5}^{2}+d_{6}^{2}\right) t}{4 c}+d_{3}\right) i
\end{aligned}
$$


by applying the transformation (1.5) to (2.42), where $b_{r} \in \mathbb{R}$.

Case 4. $\phi=\left(x^{2}+y^{2}\right) / 4 c t+\beta$ for some function $\beta$ of $t$.

Under our assumption, (2.5) becomes

$$
\xi_{t}+\frac{x}{t} \xi_{x}+\frac{y}{t} \xi_{y}+\frac{1}{t} \xi=0 .
$$

Thus we have:

$$
\xi=\frac{1}{t} \zeta(u, v), \quad u=\frac{x}{t}, v=\frac{y}{t},
$$

for some two-variable function $\zeta$. Moreover, (2.6) becomes

$$
-\beta^{\prime} \zeta+\frac{c}{t^{2}}\left(\zeta_{u u}+\zeta_{v v}\right)+\frac{a}{t^{2}} \zeta^{3}=0
$$

An obvious solution is

$$
\zeta=d, \quad \beta=-\frac{a d^{2}}{t}, \quad d \in \mathbb{R} .
$$

If $a c<0$, we have the simple following solutions with $\beta=0$ :

$$
\zeta=\frac{1}{\ell_{1} u+\ell_{2} v+\ell_{3}} \sqrt{-\frac{2 c\left(\ell_{1}^{2}+\ell_{2}^{2}\right)}{a}} \quad \text { or } \quad \sqrt{-\frac{c}{a\left(\left(u-\ell_{1}\right)^{2}+\left(v-\ell_{2}\right)^{2}\right)}}
$$

for $\ell_{1}, \ell_{2}, \ell_{3} \in \mathbb{R}$.

Next we assume

$$
\beta^{\prime}=\frac{b}{t^{2}} \Longrightarrow \beta=-\frac{b}{t}
$$

modulo the transformation in (1.2), where $b$ is a real constant to be determined. Suppose

$$
\zeta=\Im(\varpi), \quad \varpi=\ell_{1} u+\ell_{2} v+\ell_{3}
$$

for $\ell_{1}, \ell_{2}, \ell_{3} \in \mathbb{R}$ such that $\left(\ell_{1}, \ell_{2}\right) \neq(0,0)$. Then $(2.46)$ is equivalent to:

$$
-b \Im+c\left(\ell_{1}^{2}+\ell_{2}^{2}\right) \Im^{\prime \prime}+a \Im^{3}=0 .
$$

According to (2.10), (2.11) and (2.13)-(2.15), we have the following solutions: If $a c<0$, we have the following solutions:

$$
\begin{aligned}
& \Im=\sqrt{-\frac{2 c\left(\ell_{1}^{2}+\ell_{2}^{2}\right)}{a}} \tan \varpi, \quad b=2 c\left(\ell_{1}^{2}+\ell_{2}^{2}\right) ; \\
& \Im=\sqrt{-\frac{2 c\left(\ell_{1}^{2}+\ell_{2}^{2}\right)}{a}} \sec \varpi, \quad b=-c\left(\ell_{1}^{2}+\ell_{2}^{2}\right) ; \\
& \Im=\sqrt{-\frac{2 c\left(\ell_{1}^{2}+\ell_{2}^{2}\right)}{a}} \operatorname{coth} \varpi, \quad b=-2 c\left(\ell_{1}^{2}+\ell_{2}^{2}\right) ;
\end{aligned}
$$




$$
\begin{gathered}
\Im=\sqrt{-\frac{2 c\left(\ell_{1}^{2}+\ell_{2}^{2}\right)}{a}} \operatorname{csch} \varpi, \quad b=c\left(\ell_{1}^{2}+\ell_{2}^{2}\right) ; \\
\Im=m \sqrt{-\frac{2 c\left(\ell_{1}^{2}+\ell_{2}^{2}\right)}{a}} \operatorname{sn} \varpi, \quad b=-\left(1+m^{2}\right) c\left(\ell_{1}^{2}+\ell_{2}^{2}\right) .
\end{gathered}
$$

When $a c>0$, we get the following solutions:

$$
\begin{array}{cl}
\Im=m \sqrt{\frac{2 c\left(\ell_{1}^{2}+\ell_{2}^{2}\right)}{a}} \text { cn } \varpi, & b=\left(2 m^{2}-1\right) c\left(\ell_{1}^{2}+\ell_{2}^{2}\right), \\
\Im=\sqrt{\frac{2 c\left(\ell_{1}^{2}+\ell_{2}^{2}\right)}{a}} \operatorname{dn} \varpi, & b=\left(2-m^{2}\right) c\left(\ell_{1}^{2}+\ell_{2}^{2}\right) .
\end{array}
$$

Theorem 2.5. Let $\ell_{1}, \ell_{2}, \ell_{3}, \ell_{4}, \ell_{5}, m \in \mathbb{R}$ such that $\left(\ell_{1}, \ell_{2}\right) \neq(0,0)$. The following functions are solutions $\psi$ of the two-dimensional cubic nonlinear cubic nonlinear Schrödinger equation: if ac $<0$,

$$
\begin{aligned}
& \frac{1}{\ell_{1} x+\ell_{2} y+\ell_{3} t} \sqrt{-\frac{2 c\left(\ell_{1}^{2}+\ell_{2}^{2}\right)}{a}} e^{\left(x^{2}+y^{2}\right) i / 4 c t}, \\
& \sqrt{-\frac{c}{a\left(\left(x-\ell_{4} t\right)^{2}+\left(y-\ell_{5} t\right)^{2}\right)}} e^{\left(x^{2}+y^{2}\right) i / 4 c t}, \\
& \sqrt{-\frac{2 c\left(\ell_{1}^{2}+\ell_{2}^{2}\right)}{a}} \frac{1}{t} \tan \frac{\ell_{1} x+\ell_{2} y+\ell_{3} t}{t} \exp \left(\frac{x^{2}+y^{2}}{4 c t}-\frac{2 c\left(\ell_{1}^{2}+\ell_{2}^{2}\right)}{t}\right) i, \\
& \sqrt{-\frac{2 c\left(\ell_{1}^{2}+\ell_{2}^{2}\right)}{a}} \frac{1}{t} \sec \frac{\ell_{1} x+\ell_{2} y+\ell_{3} t}{t} \exp \left(\frac{x^{2}+y^{2}}{4 c t}+\frac{c\left(\ell_{1}^{2}+\ell_{2}^{2}\right)}{t}\right) i \text {, } \\
& \sqrt{-\frac{2 c\left(\ell_{1}^{2}+\ell_{2}^{2}\right)}{a}} \frac{1}{t} \operatorname{coth} \frac{\ell_{1} x+\ell_{2} y+\ell_{3} t}{t} \exp \left(\frac{x^{2}+y^{2}}{4 c t}+\frac{2 c\left(\ell_{1}^{2}+\ell_{2}^{2}\right)}{t}\right) i \text {, } \\
& \sqrt{-\frac{2 c\left(\ell_{1}^{2}+\ell_{2}^{2}\right)}{a}} \frac{1}{t} \operatorname{csch} \frac{\ell_{1} x+\ell_{2} y+\ell_{3} t}{t} \exp \left(\frac{x^{2}+y^{2}}{4 c t}-\frac{c\left(\ell_{1}^{2}+\ell_{2}^{2}\right)}{t}\right) i \\
& m \sqrt{-\frac{2 c\left(\ell_{1}^{2}+\ell_{2}^{2}\right)}{a}} \frac{1}{t} \operatorname{sn} \frac{\ell_{1} x+\ell_{2} y+\ell_{3} t}{t} \exp \left(\frac{x^{2}+y^{2}}{4 c t}+\frac{c\left(1+m^{2}\right)\left(\ell_{1}^{2}+\ell_{2}^{2}\right)}{t}\right) i \text {; }
\end{aligned}
$$

when $a c>0$,

$$
\begin{gathered}
m \sqrt{\frac{2 c\left(\ell_{1}^{2}+\ell_{2}^{2}\right)}{a}} \frac{1}{t} \operatorname{cn} \frac{\ell_{1} x+\ell_{2} y+\ell_{3} t}{t} \exp \left(\frac{x^{2}+y^{2}}{4 c t}-\frac{c\left(2 m^{2}-1\right)\left(\ell_{1}^{2}+\ell_{2}^{2}\right)}{t}\right) i \\
\sqrt{\frac{2 c\left(\ell_{1}^{2}+\ell_{2}^{2}\right)}{a}} \frac{1}{t} \operatorname{dn} \frac{\ell_{1} x+\ell_{2} y+\ell_{3} t}{t} \exp \left(\frac{x^{2}+y^{2}}{4 c t}-\frac{c\left(2-m^{2}\right)\left(\ell_{1}^{2}+\ell_{2}^{2}\right)}{t}\right) i .
\end{gathered}
$$


Remark 2.6. Applying the transformation (1.5) to the solution (2.59), we get another solution:

$$
\psi=\frac{e^{\left[2\left(d_{1} x+d_{3} y\right)+\left(d_{1}^{2}+d_{3}^{2}\right) t\right] i / 4 c \sqrt{-\frac{2 c\left(\ell_{1}^{2}+\ell_{2}^{2}\right)}{a}}}}{\ell_{1}\left(x-d_{1} t+d_{2}\right)+\ell_{2}\left(y-d_{3} t+d_{4}\right)+\ell_{3} t} e^{\left(\left(x-d_{1} t+d_{2}\right)^{2}+\left(y-d_{3} t+d_{4}\right)^{2}\right) i / 4 c t},
$$

where $d_{1}, d_{2}, d_{3}, d_{4} \in \mathbb{R}$.

\section{Exact Solutions of the Coupled Equations}

In this section, we will use our results in previous section to find exact solutions of the coupled two-dimensional cubic nonlinear Schrödinger equations (1.2) and (1.3).

Write

$$
\psi=\xi(t, x, y) e^{i \phi(t, x, y)}, \quad \varphi=\eta(t, x, y) e^{i \mu(t, x, y)}
$$

where $\xi, \phi, \eta$ and $\mu$ are real functions in $t, x, y$. As the arguments in (2.1)-(2.6), the system (1.2) and (1.3) is equivalent to the following system for real functions:

$$
\begin{gathered}
\xi_{t}+c_{1}\left(2 \xi_{x} \phi_{x}+2 \xi_{y} \phi_{y}+\xi\left(\phi_{x x}+\phi_{y y}\right)\right)=0 \\
-\xi\left[\phi_{t}+c_{1}\left(\phi_{x}^{2}+\phi_{y}^{2}\right)\right]+c_{1}\left(\xi_{x x}+\xi_{y y}\right)+\left(a_{1} \xi^{2}+b_{1} \eta^{2}\right) \xi=0 \\
\eta_{t}+c_{2}\left(2 \eta_{x} \mu_{x}+2 \eta_{y} \mu_{y}+\eta\left(\mu_{x x}+\mu_{y y}\right)\right)=0, \\
-\eta\left[\mu_{t}+c_{2}\left(\mu_{x}^{2}+\mu_{y}^{2}\right)\right]+c_{2}\left(\eta_{x x}+\eta_{y y}\right)+\left(a_{2} \xi^{2}+b_{2} \eta^{2}\right) \eta=0 .
\end{gathered}
$$

Based on our experience in last section, we will solve the above system according to the following cases. For the convenience, we always assume the conditions on the constants involved in an expression such that it make sense. For instance, when we use $\sqrt{d_{1}-d_{2}}$, we naturally assume $d_{1} \geq d_{2}$.

Case 1. $(\phi, \mu)=(0,0)$ and $a_{1} b_{2}-a_{2} b_{1} \neq 0$.

In this case, $\xi_{t}=\eta_{t}=0$ by (3.2) and (3.4). Moreover, (3.3) and (3.5) become

$$
c_{1}\left(\xi_{x x}+\xi_{y y}\right)+\left(a_{1} \xi^{2}+b_{1} \eta^{2}\right) \xi=0, \quad c_{2}\left(\eta_{x x}+\eta_{y y}\right)+\left(a_{2} \xi^{2}+b_{2} \eta^{2}\right) \eta=0
$$

Assume

$$
\xi=\frac{\iota_{1}}{x}, \quad \eta=\frac{\iota_{2}}{x}
$$

Then (3.6) is equivalent to:

$$
a_{1} \iota_{1}^{2}+b_{1} \iota_{2}^{2}+2 c_{1}=0, \quad a_{2} \iota^{2}+b_{2} \iota^{2}+2 c_{2}=0 .
$$


Solving the above linear algebraic equations for $\iota_{1}^{2}$ and $\iota_{2}^{2}$, we have:

$$
\iota_{1}^{2}=\frac{2\left(b_{1} c_{2}-b_{2} c_{1}\right)}{a_{1} b_{2}-a_{2} b_{1}}, \quad \iota_{2}^{2}=\frac{2\left(a_{2} c_{1}-a_{1} c_{2}\right)}{a_{1} b_{2}-a_{2} b_{1}} .
$$

Thus we have the following solution

$$
\xi=\frac{\epsilon_{1}}{x} \sqrt{\frac{2\left(b_{1} c_{2}-b_{2} c_{1}\right)}{a_{1} b_{2}-a_{2} b_{1}}}, \quad \eta=\frac{\epsilon_{2}}{x} \sqrt{\frac{2\left(a_{2} c_{1}-a_{1} c_{2}\right)}{a_{1} b_{2}-a_{2} b_{1}}}
$$

for $\epsilon_{1}, \epsilon_{2} \in\{1,-1\}$. Similarly, we have the solution:

$$
\xi=\epsilon_{1} \sqrt{\frac{b_{1} c_{2}-b_{2} c_{1}}{\left(a_{1} b_{2}-a_{2} b_{1}\right)\left(x^{2}+y^{2}\right)}}, \quad \eta=\epsilon_{2} \sqrt{\frac{a_{2} c_{1}-a_{1} c_{2}}{\left(a_{1} b_{2}-a_{2} b_{1}\right)\left(x^{2}+y^{2}\right)}} .
$$

Case 2. $(\phi, \mu)=\left(k_{1} t, k_{2} t\right)$ with $k_{1}, k_{2} \in \mathbb{R}$.

Again we have $\xi_{t}=\eta_{t}=0$ by (3.2) and (3.4). Moreover, (3.3) and (3.5) become

$$
-k_{1} \xi+c_{1}\left(\xi_{x x}+\xi_{y y}\right)+\left(a_{1} \xi^{2}+b_{1} \eta^{2}\right) \xi=0, \quad-k_{2} \eta+c_{2}\left(\eta_{x x}+\eta_{y y}\right)+\left(a_{2} \xi^{2}+b_{2} \eta^{2}\right) \eta=0
$$

First we assume $a_{1} b_{2}-a_{2} b_{1} \neq 0$ and

$$
\xi=\iota_{1} \Im(x), \quad \eta=\iota_{2} \Im(x) .
$$

Then (3.12) becomes

$$
-k_{1} \Im+c_{1} \Im^{\prime \prime}+\left(a_{1} \iota_{1}^{2}+b_{1} \iota_{2}^{2}\right) \Im^{3}=0, \quad-k_{2} \Im+c_{2} \Im^{\prime \prime}+\left(a_{2} \iota^{2}+b_{2} \iota^{2}\right) \Im^{3}=0 .
$$

According to (2.10) and (2.11), when $\Im=\tan x, \sec x, \operatorname{coth} x$ and $\operatorname{csch} x$, we always have

$$
a_{1} \iota_{1}^{2}+b_{1} \iota_{2}^{2}+2 c_{1}=0, \quad a_{2} \iota^{2}+b_{2} \iota^{2}+2 c_{2}=0
$$

Thus for $\epsilon_{1}, \epsilon_{2} \in\{1,-1\}$, we have the following solutions:

$$
\begin{gathered}
\xi=\epsilon_{1} \sqrt{\frac{2\left(b_{1} c_{2}-b_{2} c_{1}\right)}{a_{1} b_{2}-a_{2} b_{1}}} \tan x, \quad \eta=\epsilon_{2} \sqrt{\frac{2\left(a_{2} c_{1}-a_{1} c_{2}\right)}{a_{1} b_{2}-a_{2} b_{1}}} \tan x, \quad\left(k_{1}, k_{2}\right)=2\left(c_{1}, c_{2}\right) \\
\xi=\epsilon_{1} \sqrt{\frac{2\left(b_{1} c_{2}-b_{2} c_{1}\right)}{a_{1} b_{2}-a_{2} b_{1}}} \sec x, \quad \eta=\epsilon_{2} \sqrt{\frac{2\left(a_{2} c_{1}-a_{1} c_{2}\right)}{a_{1} b_{2}-a_{2} b_{1}}} \sec x, \quad\left(k_{1}, k_{2}\right)=-\left(c_{1}, c_{2}\right) ; \\
\xi=\epsilon_{1} \sqrt{\frac{2\left(b_{1} c_{2}-b_{2} c_{1}\right)}{a_{1} b_{2}-a_{2} b_{1}}} \operatorname{coth} x, \quad \eta=\epsilon_{2} \sqrt{\frac{2\left(a_{2} c_{1}-a_{1} c_{2}\right)}{a_{1} b_{2}-a_{2} b_{1}}} \operatorname{coth} x
\end{gathered}
$$


and $\left(k_{1}, k_{2}\right)=-2\left(c_{1}, c_{2}\right)$;

$$
\xi=\epsilon_{1} \sqrt{\frac{2\left(b_{1} c_{2}-b_{2} c_{1}\right)}{a_{1} b_{2}-a_{2} b_{1}}} \operatorname{csch} x, \quad \eta=\epsilon_{2} \sqrt{\frac{2\left(a_{2} c_{1}-a_{1} c_{2}\right)}{a_{1} b_{2}-a_{2} b_{1}}} \operatorname{csch} x, \quad\left(k_{1}, k_{2}\right)=\left(c_{1}, c_{2}\right) .
$$

Similarly, (2.13)-(2.15) give us the following solutions:

$$
\xi=m \epsilon_{1} \sqrt{\frac{2\left(b_{1} c_{2}-b_{2} c_{1}\right)}{a_{1} b_{2}-a_{2} b_{1}}} \text { sn } x, \quad \eta=m \epsilon_{2} \sqrt{\frac{2\left(a_{2} c_{1}-a_{1} c_{2}\right)}{a_{1} b_{2}-a_{2} b_{1}}} \text { sn } x
$$

and $\left(k_{1}, k_{2}\right)=-\left(1+m^{2}\right)\left(c_{1}, c_{2}\right)$;

$$
\xi=m \epsilon_{1} \sqrt{\frac{2\left(b_{2} c_{1}-b_{1} c_{2}\right)}{a_{1} b_{2}-a_{2} b_{1}}} \text { cn } x \quad \eta=m \epsilon_{2} \sqrt{\frac{2\left(a_{1} c_{2}-a_{2} c_{1}\right)}{a_{1} b_{2}-a_{2} b_{1}}} \text { cn } x
$$

and $\left(k_{1}, k_{2}\right)=\left(2 m^{2}-1\right)\left(c_{1}, c_{2}\right)$;

$$
\xi=\epsilon_{1} \sqrt{\frac{2\left(b_{2} c_{1}-b_{1} c_{2}\right)}{a_{1} b_{2}-a_{2} b_{1}}} \operatorname{dn} x, \quad \eta=\epsilon_{2} \sqrt{\frac{2\left(a_{1} c_{2}-a_{2} c_{1}\right)}{a_{1} b_{2}-a_{2} b_{1}}} \operatorname{dn} x
$$

and $\left(k_{1}, k_{2}\right)=\left(2-m^{2}\right)\left(c_{1}, c_{2}\right)$.

If $\left(a_{1}, b_{1}\right)=a_{1}\left(1, d^{2}\right)$ and $\left(a_{2}, b_{2}\right)=a_{2}\left(1, d^{2}\right)$ with $d \in \mathbb{R}$, we have the following solution of $(3.12)$ :

$$
\xi=d \ell \sin x, \quad \eta=\ell \cos x, \quad\left(k_{1}, k_{2}\right)=\left(a_{1}(d \ell)^{2}-c_{1}, a_{2}(d \ell)^{2}-c_{2}\right)
$$

for $\ell \in \mathbb{R}$. When $\left(a_{1}, b_{1}\right)=a_{1}\left(1,-d^{2}\right)$ and $\left(a_{2}, b_{2}\right)=a_{2}\left(1,-d^{2}\right)$ with $d \in \mathbb{R}$, we get the solution:

$$
\xi=d \ell \cosh x, \quad \eta=\ell \sinh x, \quad\left(k_{1}, k_{2}\right)=\left(a_{1}(d \ell)^{2}+c_{1}, a_{2}(d \ell)^{2}+c_{2}\right) .
$$

In summary, we have the following theorem.

Theorem 3.1. Let $d, \ell, m \in \mathbb{R}$ and let $\epsilon_{1}, \epsilon_{2} \in\{1,-1\}$. If $a_{1} b_{2}-a_{2} b_{1} \neq 0$, we have the following solutions of the coupled two-dimensional cubic nonlinear Schrödinger equations (1.2) and (1.3):

$$
\begin{array}{r}
\psi=\frac{\epsilon_{1}}{x} \sqrt{\frac{2\left(b_{1} c_{2}-b_{2} c_{1}\right)}{a_{1} b_{2}-a_{2} b_{1}}}, \quad \varphi=\frac{\epsilon_{2}}{x} \sqrt{\frac{2\left(a_{2} c_{1}-a_{1} c_{2}\right)}{a_{1} b_{2}-a_{2} b_{1}}} ; \\
\psi=\epsilon_{1} \sqrt{\frac{b_{1} c_{2}-b_{2} c_{1}}{\left(a_{1} b_{2}-a_{2} b_{1}\right)\left(x^{2}+y^{2}\right)}}, \quad \varphi=\epsilon_{2} \sqrt{\frac{a_{2} c_{1}-a_{1} c_{2}}{\left(a_{1} b_{2}-a_{2} b_{1}\right)\left(x^{2}+y^{2}\right)}} ; \\
\psi=\epsilon_{1} \sqrt{\frac{2\left(b_{1} c_{2}-b_{2} c_{1}\right)}{a_{1} b_{2}-a_{2} b_{1}}} e^{2 c_{1} t i} \tan x, \quad \varphi=\epsilon_{2} \sqrt{\frac{2\left(a_{2} c_{1}-a_{1} c_{2}\right)}{a_{1} b_{2}-a_{2} b_{1}}} e^{2 c_{2} t i} \tan x ;
\end{array}
$$




$$
\begin{aligned}
& \psi=\epsilon_{1} \sqrt{\frac{2\left(b_{1} c_{2}-b_{2} c_{1}\right)}{a_{1} b_{2}-a_{2} b_{1}}} e^{-c_{1} t i} \sec x, \quad \varphi=\epsilon_{2} \sqrt{\frac{2\left(a_{2} c_{1}-a_{1} c_{2}\right)}{a_{1} b_{2}-a_{2} b_{1}}} e^{-c_{2} t i} \sec x ; \\
& \psi=\epsilon_{1} \sqrt{\frac{2\left(b_{1} c_{2}-b_{2} c_{1}\right)}{a_{1} b_{2}-a_{2} b_{1}}} e^{-2 c_{1} t i} \operatorname{coth} x, \quad \varphi=\epsilon_{2} \sqrt{\frac{2\left(a_{2} c_{1}-a_{1} c_{2}\right)}{a_{1} b_{2}-a_{2} b_{1}}} e^{-2 c_{2} t i} \operatorname{coth} x ; \\
& \psi=\epsilon_{1} \sqrt{\frac{2\left(b_{1} c_{2}-b_{2} c_{1}\right)}{a_{1} b_{2}-a_{2} b_{1}}} e^{c_{1} t i} \operatorname{csch} x, \quad \varphi=\epsilon_{2} \sqrt{\frac{2\left(a_{2} c_{1}-a_{1} c_{2}\right)}{a_{1} b_{2}-a_{2} b_{1}}} e^{c_{2} t i} \operatorname{csch} x ; \\
& \psi=m \epsilon_{1} \sqrt{\frac{2\left(b_{1} c_{2}-b_{2} c_{1}\right)}{a_{1} b_{2}-a_{2} b_{1}}} e^{-\left(1+m^{2}\right) c_{1} t i} \operatorname{sn} x, \\
& \varphi=m \epsilon_{2} \sqrt{\frac{2\left(a_{2} c_{1}-a_{1} c_{2}\right)}{a_{1} b_{2}-a_{2} b_{1}}} e^{-\left(1+m^{2}\right) c_{2} t i} \operatorname{sn} x ; \\
& \psi=m \epsilon_{1} \sqrt{\frac{2\left(b_{2} c_{1}-b_{1} c_{2}\right)}{a_{1} b_{2}-a_{2} b_{1}}} e^{\left(2 m^{2}-1\right) c_{1} t i} \operatorname{cn} x, \\
& \varphi=m \epsilon_{2} \sqrt{\frac{2\left(a_{1} c_{2}-a_{2} c_{1}\right)}{a_{1} b_{2}-a_{2} b_{1}}} e^{\left(2 m^{2}-1\right) c_{2} t i} \operatorname{cn} x ; \\
& \psi=\epsilon_{1} \sqrt{\frac{2\left(b_{2} c_{1}-b_{1} c_{2}\right)}{a_{1} b_{2}-a_{2} b_{1}}} e^{\left(2-m^{2}\right) c_{1} t i} \operatorname{dn} x, \quad \varphi=\epsilon_{2} \sqrt{\frac{2\left(a_{1} c_{2}-a_{2} c_{1}\right)}{a_{1} b_{2}-a_{2} b_{1}}} e^{\left(2-m^{2}\right) c_{1} t i} \operatorname{dn} x . \\
& \text { If }\left(a_{1}, b_{1}\right)=a_{1}\left(1, d^{2}\right) \text { and }\left(a_{2}, b_{2}\right)=a_{2}\left(1, d^{2}\right) \text {, } \\
& \psi=d \ell e^{\left(a_{1}(d \ell)^{2}-c_{1}\right) t i} \sin x, \quad \varphi=\ell e^{\left(a_{2}(d \ell)^{2}-c_{2}\right) t i} \cos x .
\end{aligned}
$$

When $\left(a_{1}, b_{1}\right)=a_{1}\left(1,-d^{2}\right)$ and $\left(a_{2}, b_{2}\right)=a_{2}\left(1,-d^{2}\right)$,

$$
\psi=d \ell e^{\left(a_{1}(d \ell)^{2}+c_{1}\right) t i} \cosh x, \quad \eta=\ell e^{\left(a_{2}(d \ell)^{2}+c_{2}\right) t i} \sinh x .
$$

Remark 3.2. Applying the symmetric transformations (1.6)-(1.9) to the above solutions, we can get more sophisticated ones. For instance, applying $T_{1}$ in (1.6) and (1.7) to (3.25) and (3.37), we get

$$
\psi=\frac{\epsilon_{1} e^{d_{2} i}}{x \cos d_{1}+y \sin d_{1}} \sqrt{\frac{2\left(b_{1} c_{2}-b_{2} c_{1}\right)}{a_{1} b_{2}-a_{2} b_{1}}}, \quad \varphi=\frac{\epsilon_{2} e^{d_{3} i}}{x \cos d_{1}+y \sin d_{1}} \sqrt{\frac{2\left(a_{2} c_{1}-a_{1} c_{2}\right)}{a_{1} b_{2}-a_{2} b_{1}}} ;
$$

and

$$
\begin{gathered}
\psi=d d_{2} \ell e^{\left[\left(a_{1}(d \ell)^{2}+c_{1}\right) d_{2}^{2} t+d_{3}\right] i} \cosh d_{2}\left(x \cos d_{1}+y \sin d_{1}\right), \\
\eta=d_{2} \ell e^{\left[\left(a_{2}(d \ell)^{2}+c_{2}\right) d_{2}^{2} t+d_{4}\right] i} \sinh d_{2}\left(x \cos d_{1}+y \sin d_{1}\right) .
\end{gathered}
$$


Applying $T_{2}$ in (1.8) and (1.9) to (3.26), we obtain:

$$
\begin{aligned}
& \psi=\epsilon_{1} e^{\left[2\left(d_{1} x+d_{3} y\right)+\left(d_{1}^{2}+d_{3}^{2}\right) t\right] i / 4 c_{1}} \sqrt{\frac{b_{1} c_{2}-b_{2} c_{1}}{\left(a_{1} b_{2}-a_{2} b_{1}\right)\left(\left(x-d_{1} t+d_{2}\right)^{2}+\left(y-d_{2} t+d_{4}\right)^{2}\right)}}, \\
& \varphi=\epsilon_{2} e^{\left[2\left(d_{1} x+d_{3} y\right)+\left(d_{1}^{2}+d_{3}^{2}\right) t\right] i / 4 c_{2}} \sqrt{\frac{a_{2} c_{1}-a_{1} c_{2}}{\left(a_{1} b_{2}-a_{2} b_{1}\right)\left(\left(x-d_{1} t+d_{2}\right)^{2}+\left(y-d_{2} t+d_{4}\right)^{2}\right)}} .
\end{aligned}
$$

Case 3. $\phi=x^{2} / 4 c_{1} t+\beta_{1}$ and $\mu=(x-d)^{2} / 4 c_{2}(t-\ell)+\beta_{2}$ or $\mu=y^{2} / 4 c_{2}(t-\ell)+\beta_{2}$ for some functions $\beta_{1}$ and $\beta_{2}$ of $t$ and real constants $d$ and $\ell$.

First we assume $\mu=(x-d)^{2} / 4 c_{2}(t-\ell)+\beta_{2}$. Then (3.2) and (3.4) become

$$
\xi_{t}+\frac{x}{t} \xi_{x}+\frac{1}{2 t} \xi=0, \quad \eta_{t}+\frac{x-d}{t-\ell} \eta_{x}+\frac{1}{2(t-\ell)} \eta=0 .
$$

Thus

$$
\xi=\frac{1}{\sqrt{t}} \hat{\xi}\left(t^{-1} x, y\right), \quad \eta=\frac{1}{\sqrt{t-\ell}} \hat{\eta}\left((t-\ell)^{-1}(x-d), y\right)
$$

for some two-variable functions $\hat{\xi}$ and $\hat{\eta}$. On the other hand, (3.3) and (3.5) become:

$$
\begin{aligned}
& -\beta_{1}^{\prime} \xi+c_{1}\left(\xi_{x x}+\xi_{y y}\right)+\left(a_{1} \xi^{2}+b_{1} \eta^{2}\right) \xi=0, \\
& -\beta_{2}^{\prime} \eta+c_{2}\left(\eta_{x x}+\eta_{y y}\right)+\left(a_{2} \xi^{2}+b_{2} \eta^{2}\right) \eta=0 .
\end{aligned}
$$

As (2.31)-(2.33), the above two equations force us to take

$$
\xi=\frac{k_{1}}{\sqrt{t}}, \quad \eta=\frac{k_{2}}{\sqrt{t-\ell}} .
$$

So (3.45) and (3.46) are implied by the equations:

$$
\beta_{1}^{\prime}=\frac{a_{1} k_{1}^{2}}{t}+\frac{b_{1} k_{2}^{2}}{t-\ell}, \quad \beta_{2}^{\prime}=\frac{a_{2} k_{1}^{2}}{t}+\frac{b_{2} k_{2}^{2}}{t-\ell} .
$$

Modulo the transformation (1.6) and (1.7), we take

$$
\beta_{1}=a_{1} k_{1}^{2} \ln t+b_{1} k_{2}^{2} \ln (t-\ell), \quad \beta_{2}=a_{2} k_{1}^{2} \ln t+b_{2} k_{2}^{2} \ln (t-\ell) .
$$

Exact same approach holds for $\mu=y^{2} / 4 c_{2}(t-\ell)+\beta_{2}$.

Theorem 3.3. Let $d, \ell, k_{1}, k_{2} \in \mathbb{R}$. We have the following solutions of the coupled two-dimensional cubic nonlinear Schrödinger equations (1.2) and (1.3):

$$
\psi=k_{1} t^{a_{1} k_{1}^{2} i-1 / 2}(t-\ell)^{b_{1} k_{2}^{2} i} e^{x^{2} i / 2 c_{1} t}, \quad \varphi=k_{2} t^{a_{2} k_{1}^{2} i}(t-\ell)^{b_{2} k_{2}^{2} i-1 / 2} e^{(x-d)^{2} i / 2 c_{2}(t-\ell)}
$$




$$
\psi=k_{1} t^{a_{1} k_{1}^{2} i-1 / 2}(t-\ell)^{b_{1} k_{2}^{2} i} e^{x^{2} i / 2 c_{1} t}, \quad \varphi=k_{2} t^{a_{2} k_{1}^{2} i}(t-\ell)^{b_{2} k_{2}^{2} i-1 / 2} e^{y^{2} i / 2 c_{2}(t-\ell)} .
$$

Case 4. $\phi=x^{2} / 4 c_{1} t+\beta_{1}$ and $\mu=(x-d)^{2} / 4 c_{2}\left(t-d_{1}\right)+y^{2} / 4 c_{2}\left(t-d_{2}\right)+\beta_{2}$ for some functions $\beta_{1}$ and $\beta_{2}$ of $t$ and real constants $d, d_{1}$ and $d_{2}$.

In this case, (3.2) and (3.4) become

$$
\xi_{t}+\frac{x}{t} \xi_{x}+\frac{1}{2 t} \xi=0, \quad \eta_{t}+\frac{x-d}{t-d_{1}} \eta_{x}+\frac{y}{t-d_{2}} \eta_{y}+\left(\frac{1}{2\left(t-d_{1}\right)}+\frac{1}{2\left(t-d_{2}\right)}\right) \xi=0 .
$$

Thus

$$
\xi=\frac{1}{\sqrt{t}} \hat{\xi}\left(t^{-1} x, y\right), \quad \eta=\frac{1}{\sqrt{\left(t-d_{1}\right)\left(t-d_{2}\right)}} \hat{\eta}\left(\left(t-d_{1}\right)^{-1}(x-d),\left(t-d_{2}\right)^{-1} y\right)
$$

for some two-variable functions $\hat{\xi}$ and $\hat{\eta}$. Again (3.3) and (3.5) become (3.45) and (3.46), respectively. Moreover, they force us to take

$$
\xi=\frac{k_{1}}{\sqrt{t}}, \quad \eta=\frac{k_{2}}{\sqrt{\left(t-d_{1}\right)\left(t-d_{2}\right)}} .
$$

So (3.3) and (3.5) are implied by the equations:

$$
\beta_{1}^{\prime}=\frac{a_{1} k_{1}^{2}}{t}+\frac{b_{1} k_{2}^{2}}{\left(t-d_{1}\right)\left(t-d_{2}\right)}, \quad \beta_{2}^{\prime}=\frac{a_{2} k_{1}^{2}}{t}+\frac{b_{2} k_{2}^{2}}{\left(t-d_{1}\right)\left(t-d_{2}\right)} .
$$

Modulo the transformation (1.6) and (1.7), we get

$$
\beta_{1}=a_{1} k_{1}^{2} \ln t+\frac{b_{1} k_{2}^{2}}{d_{2}-d_{1}} \ln \frac{t-d_{1}}{t-d_{2}}, \quad \beta_{2}=a_{2} k_{1}^{2} \ln t+\frac{b_{2} k_{2}^{2}}{d_{2}-d_{1}} \ln \frac{t-d_{1}}{t-d_{2}}
$$

if $d_{1} \neq d_{2}$, and

$$
\beta_{1}=a_{1} k_{1}^{2} \ln t-\frac{b_{1} k_{2}^{2}}{t-d_{1}}, \quad \quad \beta_{2}=a_{2} k_{1}^{2} \ln t-\frac{b_{2} k_{2}^{2}}{t-d_{1}}
$$

when $d_{1}=d_{2}$.

Theorem 3.4. Let $d_{1}, d_{2}, k_{1}, k_{2} \in \mathbb{R}$ such that $d_{1} \neq d_{2}$. We have the following solutions of the coupled two-dimensional cubic nonlinear Schrödinger equations (1.2) and (1.3):

$$
\begin{aligned}
\psi= & k_{1} t^{a_{1} k_{1}^{2} i-1 / 2}\left(t-d_{1}\right)^{b_{1} k_{2}^{2}\left(\ell_{2}-d_{1}\right)^{-1} i}\left(t-d_{2}\right)^{-b_{1} k_{2}^{2}\left(\ell_{2}-d_{1}\right)^{-1} i} e^{x^{2} i / 4 c_{1} t} \\
\varphi= & k_{2} t^{a_{2} k_{1}^{2} i}\left(t-d_{1}\right)^{b_{1} k_{2}^{2}\left(\ell_{2}-d_{1}\right)^{-1} i-1 / 2}\left(t-d_{2}\right)^{-b_{1} k_{2}^{2}\left(\ell_{2}-d_{1}\right)^{-1} i-1 / 2} \\
& \times \exp \left(\frac{(x-d)^{2} i}{4 c_{2}\left(t-d_{1}\right)}+\frac{y^{2} i}{4 c_{2}\left(t-d_{1}\right)}\right)
\end{aligned}
$$




$$
\begin{gathered}
\psi=k_{1} t^{a_{1} k_{1}^{2} i-1 / 2} \exp \left(\frac{x^{2} i}{4 c_{1} t}-\frac{b_{1} k_{2}^{2} i}{t-d_{1}}\right), \\
\varphi=\frac{k_{2} t^{a_{2} k_{1}^{2} i}}{t-d_{1}} \exp \frac{\left((x-d)^{2}+y^{2}-4 c_{2} b_{2} k_{2}^{2}\right) i}{4 c_{2}\left(t-d_{1}\right)} .
\end{gathered}
$$

Case 5. For $\ell_{1}, \ell_{2}, \ell, d_{1}, d_{2} \in \mathbb{R}$ and functions $\beta_{1}, \beta_{2}$ of $t$,

$$
\phi=\frac{x^{2}}{4 c_{1} t}+\frac{y^{2}}{4 c_{1}(t-\ell)}+\beta_{1}, \quad \mu=\frac{\left(x-d_{1}\right)^{2}}{4 c_{2}\left(t-\ell_{1}\right)}+\frac{\left(y-d_{2}\right)^{2}}{4 c_{1}\left(t-\ell_{2}\right)}+\beta_{2} .
$$

As in the above case, we get

$$
\xi=\frac{k_{1}}{\sqrt{t(t-\ell)}}, \quad \eta=\frac{k_{2}}{\sqrt{\left(t-\ell_{1}\right)\left(t-\ell_{2}\right)}} .
$$

So (3.3) and (3.5) are implied by the equations:

$$
\beta_{1}^{\prime}=\frac{a_{1} k_{1}^{2}}{t(t-\ell)}+\frac{b_{1} k_{2}^{2}}{\left(t-\ell_{1}\right)\left(t-\ell_{2}\right)}, \quad \beta_{2}^{\prime}=\frac{a_{2} k_{1}^{2}}{t}+\frac{b_{2} k_{2}^{2}}{\left(t-\ell_{1}\right)\left(t-\ell_{2}\right)} .
$$

Modulo the transformation (1.6) and (1.7), we have

$$
\beta_{1}=\frac{a_{1} k_{1}^{2}}{\ell} \ln \frac{t-\ell}{t}+\frac{b_{1} k_{2}^{2}}{\ell_{2}-\ell_{1}} \ln \frac{t-\ell_{1}}{t-\ell_{2}}, \quad \beta_{2}=\frac{a_{2} k_{1}^{2}}{\ell} \ln \frac{t-\ell}{t}+\frac{b_{2} k_{2}^{2}}{\ell_{2}-\ell_{1}} \ln \frac{t-\ell_{1}}{t-\ell_{2}}
$$

if $\ell \neq 0$ and $\ell_{1} \neq \ell_{2}$;

$$
\beta_{1}=-\frac{a_{1} k_{1}^{2}}{t}+\frac{b_{1} k_{2}^{2}}{\ell_{2}-\ell_{1}} \ln \frac{t-\ell_{1}}{t-\ell_{2}}, \quad \beta_{2}=-\frac{a_{2} k_{1}^{2}}{t}+\frac{b_{2} k_{2}^{2}}{\ell_{2}-\ell_{1}} \ln \frac{t-\ell_{1}}{t-\ell_{2}}
$$

when $\ell=0$ and $\ell_{1} \neq \ell_{2}$;

$$
\beta_{1}=\frac{a_{1} k_{1}^{2}}{t}-\frac{b_{1} k_{2}^{2}}{t-\ell_{1}},, \quad \beta_{2}=\frac{a_{2} k_{1}^{2}}{t} t-\frac{b_{2} k_{2}^{2}}{t-\ell_{1}}
$$

if $\ell=0$ and $\ell_{1}=\ell_{2}$. Therefore, we obtain:

Theorem 3.5. Let $\ell_{1}, \ell_{2}, \ell, d_{1}, d_{2}, k_{1}, k_{2} \in \mathbb{R}$ such that $\ell \neq 0$ and $\ell_{1} \neq \ell_{2}$. We have the following solutions of the coupled two-dimensional cubic nonlinear Schrödinger equations (1.2) and (1.3):

$$
\begin{gathered}
\psi=\frac{k_{1}}{t} \exp \left(\frac{\left(x^{2}+y^{2}-4 c_{1} a_{1} k_{1}^{2}\right) i}{4 c_{1} t}-\frac{b_{1} k_{2}^{2} i}{t-\ell_{1}}\right) \\
\varphi=\frac{k_{2}}{t-\ell_{1}} \exp \left(\frac{\left(\left(x-d_{1}\right)^{2}+\left(y-d_{2}\right)^{2}-4 c_{2} b_{2} k_{2}^{2}\right) i}{4 c_{2}\left(t-\ell_{1}\right)}-\frac{a_{2} k_{1}^{2} i}{t}\right) \\
\psi=\frac{k_{1}\left(t-\ell_{1}\right)^{b_{1} k_{2}^{2}\left(\ell_{2}-\ell_{1}\right)^{-1} i}\left(t-\ell_{2}\right)^{-b_{1} k_{2}^{2}\left(\ell_{2}-\ell_{1}\right)^{-1} i}}{t} \exp \frac{\left(x^{2}+y^{2}-4 c_{1} a_{1} k_{1}^{2}\right) i}{4 c_{1} t}
\end{gathered}
$$




$$
\begin{aligned}
& \varphi=k_{2}\left(t-\ell_{1}\right)^{b_{2} k_{2}^{2}\left(\ell_{2}-\ell_{1}\right)^{-1} i-1 / 2}\left(t-\ell_{2}\right)^{-b_{2} k_{2}^{2}\left(\ell_{2}-\ell_{1}\right)^{-1} i-1 / 2} \\
& \times \exp \left(\frac{\left(x-d_{1}\right)^{2} i}{4 c_{2}\left(t-\ell_{1}\right)}+\frac{\left(y-d_{2}\right)^{2} i}{4 c_{2}\left(t-\ell_{2}\right)}-\frac{a_{2} k_{1}^{2} i}{t}\right) ; \\
& \psi=k_{1} t^{-a_{1} k_{1}^{2} \ell^{-1} i-1 / 2}(t-\ell)^{a_{1} k_{1}^{2} \ell^{-1} i-1 / 2}\left(t-\ell_{1}\right)^{b_{1} k_{2}^{2}\left(\ell_{2}-\ell_{1}\right)^{-1} i} \\
& \times\left(t-\ell_{2}\right)^{-b_{1} k_{2}^{2}\left(\ell_{2}-\ell_{1}\right)^{-1} i} \exp \left(\frac{x^{2} i}{4 c_{1} t}+\frac{y^{2} i}{4 c_{1}(t-\ell)}\right), \\
& \varphi=k_{2} t^{-a_{2} k_{1}^{2} \ell^{-1} i}(t-\ell)^{a_{2} k_{1}^{2} \ell^{-1} i}\left(t-\ell_{1}\right)^{b_{2} k_{2}^{2}\left(\ell_{2}-\ell_{1}\right)^{-1} i-1 / 2} \\
& \times\left(t-\ell_{2}\right)^{-b_{2} k_{2}^{2}\left(\ell_{2}-\ell_{1}\right)^{-1} i-1 / 2} \exp \left(\frac{\left(x-d_{1}\right)^{2} i}{4 c_{2}\left(t-\ell_{1}\right)}+\frac{\left(y-d_{2}\right)^{2} i}{4 c_{2}\left(t-\ell_{2}\right)}\right) .
\end{aligned}
$$

Case 6. For two functions $\beta_{1}, \beta_{2}$ of $t$,

$$
\phi=\frac{x^{2}+y^{2}}{4 c_{1} t}+\beta_{1}, \quad \mu=\frac{x^{2}+y^{2}}{4 c_{2} t}+\beta_{2} .
$$

As in Case 4, (3.2) and (3.4) imply

$$
\xi=\frac{1}{t} \hat{\xi}(u, v), \quad \eta=\frac{1}{t} \hat{\eta}(u, v), \quad u=\frac{x}{t}, v=\frac{y}{t} .
$$

Moreover, (3.3) and (3.5) become

$$
\begin{aligned}
& -\beta_{1}^{\prime} \hat{\xi}+\frac{c_{1}}{t^{2}}\left(\hat{\xi}_{u u}+\hat{\xi}_{v v}\right)+\frac{1}{t^{2}}\left(a_{1} \hat{\xi}^{2}+b_{1} \hat{\eta}^{2}\right) \hat{\xi}=0, \\
& -\beta_{2}^{\prime} \hat{\eta}+\frac{c_{2}}{t^{2}}\left(\hat{\eta}_{u u}+\hat{\eta}_{v v}\right)+\frac{1}{t^{2}}\left(a_{2} \hat{\xi}^{2}+b_{2} \hat{\eta}^{2}\right) \hat{\eta}=0 .
\end{aligned}
$$

To solve the above system, we assume

$$
\beta_{1}=-\frac{k_{1}}{t}, \quad \beta_{2}=-\frac{k_{2}}{t}, \quad k_{1}, k_{2} \in \mathbb{R} .
$$

Then (3.77) and (3.78) are equivalent to:

$$
\begin{aligned}
& -k_{1} \hat{\xi}+c_{1}\left(\hat{\xi}_{u u}+\hat{\xi}_{v v}\right)+\left(a_{1} \hat{\xi}^{2}+b_{1} \hat{\eta}^{2}\right) \hat{\xi}=0, \\
& -k_{2} \hat{\eta}+c_{2}\left(\hat{\eta}_{u u}+\hat{\eta}_{v v}\right)+\left(a_{2} \hat{\xi}^{2}+b_{2} \hat{\eta}^{2}\right) \hat{\eta}=0 .
\end{aligned}
$$

For $\ell_{1}, \ell_{2}, \ell_{3} \in \mathbb{R}$, we set

$$
\varpi=\ell_{1} u+\ell_{2} v+\ell_{3} .
$$

If $\left(a_{1}, b_{1}\right)=a_{1}\left(1, d^{2}\right)$ and $\left(a_{2}, b_{2}\right)=a_{2}\left(1, d^{2}\right)$ with $d \in \mathbb{R}$, we have the following solution:

$$
\hat{\xi}=d \ell \sin \varpi, \quad \hat{\eta}=\ell \cos \varpi, \quad\left(k_{1}, k_{2}\right)=\left(a_{1}(d \ell)^{2}-c_{1}\left(\ell_{1}^{2}+\ell_{2}^{2}\right), a_{2}(d \ell)^{2}-c_{2}\left(\ell_{1}^{2}+\ell_{2}^{2}\right)\right)
$$


for $\ell \in \mathbb{R}$. When $\left(a_{1}, b_{1}\right)=a_{1}\left(1,-d^{2}\right)$ and $\left(a_{2}, b_{2}\right)=a_{2}\left(1,-d^{2}\right)$ with $d \in \mathbb{R}$, we get the solution:

$$
\hat{\xi}=d \ell \cosh \varpi, \quad \hat{\eta}=\ell \sinh \varpi, \quad\left(k_{1}, k_{2}\right)=\left(c_{1}\left(\ell_{1}^{2}+\ell_{2}^{2}\right)+a_{1}(d \ell)^{2}, c_{2}\left(\ell_{1}^{2}+\ell_{2}^{2}\right)+a_{2}(d \ell)^{2}\right)
$$

for $\ell \in \mathbb{R}$.

Theorem 3.6. For $d, \ell, \ell_{1}, \ell_{2}, \ell_{3} \in \mathbb{R}$, we have the following solutions of the coupled two-dimensional cubic nonlinear Schrödinger equations (1.2) and (1.3):

$$
\begin{aligned}
& \psi=\frac{d \ell \sin \frac{\ell_{1} x+\ell_{2} y+\ell_{3} t}{t}}{t} \exp \left(\frac{x^{2}+y^{2}}{4 c_{1} t}+\frac{c_{1}\left(\ell_{1}^{2}+\ell_{2}^{2}\right)-a_{1}(d \ell)^{2}}{t}\right) i \\
& \varphi=\frac{\ell \cos \frac{\ell_{1} x+\ell_{2} y+\ell_{3} t}{t}}{t} \exp \left(\frac{x^{2}+y^{2}}{4 c_{2} t}+\frac{c_{2}\left(\ell_{1}^{2}+\ell_{2}^{2}\right)-a_{2}(d \ell)^{2}}{t}\right) i
\end{aligned}
$$

if $\left(a_{1}, b_{1}\right)=a_{1}\left(1, d^{2}\right)$ and $\left(a_{2}, b_{2}\right)=a_{2}\left(1, d^{2}\right)$;

$$
\begin{gathered}
\psi=\frac{d \ell \cosh \frac{\ell_{1} x+\ell_{2} y+\ell_{3} t}{t}}{t} \exp \left(\frac{x^{2}+y^{2}}{4 c_{1} t}-\frac{c_{1}\left(\ell_{1}^{2}+\ell_{2}^{2}\right)+a_{1}(d \ell)^{2}}{t}\right) i \\
\varphi=\frac{\ell \sinh \frac{\ell_{1} x+\ell_{2} y+\ell_{3} t}{t}}{t} \exp \left(\frac{x^{2}+y^{2}}{4 c_{2} t}-\frac{c_{2}\left(\ell_{1}^{2}+\ell_{2}^{2}\right)+a_{2}(d \ell)^{2}}{t}\right) i
\end{gathered}
$$

when $\left(a_{1}, b_{1}\right)=a_{1}\left(1,-d^{2}\right)$ and $\left(a_{2}, b_{2}\right)=a_{2}\left(1,-d^{2}\right)$.

Finally, we assume $a_{1} b_{2}-a_{2} b_{1} \neq 0$. Recall the notion in (3.81). Taking $k_{1}=k_{2}=0$, the we have the following solutions of the system of (3.79) and (3.80):

$$
\hat{\xi}=\frac{1}{\varpi} \sqrt{\frac{2\left(b_{1} c_{2}-b_{2} c_{1}\right)\left(\ell_{1}^{2}+\ell_{2}^{2}\right)}{a_{1} b_{2}-a_{2} b_{1}}}, \quad \hat{\eta}=\frac{1}{\varpi} \sqrt{\frac{2\left(a_{2} c_{1}-a_{1} c_{2}\right)\left(\ell_{1}^{2}+\ell_{2}^{2}\right)}{a_{1} b_{2}-a_{2} b_{1}}}
$$

and

$$
\begin{aligned}
& \hat{\xi}=\sqrt{\frac{b_{1} c_{2}-b_{2} c_{1}}{\left(a_{1} b_{2}-a_{2} b_{1}\right)\left(\left(u-d_{1}\right)^{2}+\left(v-d_{2}\right)^{2}\right)}}, \\
& \hat{\eta}=\sqrt{\frac{a_{2} c_{1}-a_{1} c_{2}}{\left(a_{1} b_{2}-a_{2} b_{1}\right)\left(\left(u-d_{1}\right)^{2}+\left(v-d_{2}\right)^{2}\right)}}
\end{aligned}
$$

for $\ell_{1}, \ell_{2} \in \mathbb{R}$. In general, we assume

$$
\hat{\xi}=\iota_{1} \Im(\varpi), \quad \hat{\eta}=\iota_{2} \Im(\varpi)
$$

for some one-variable function $\Im$ and $k_{1}, k_{2} \in \mathbb{R}$. Then (3.79) and (3.80) implied by

$$
\begin{aligned}
& -k_{1} \Im+c_{1}\left(\ell_{1}^{2}+\ell_{2}^{2}\right) \Im^{\prime \prime}+\left(a_{1} \iota_{1}^{2}+b_{1} \iota_{2}^{2}\right) \Im^{3}=0, \\
& -k_{2} \Im+c_{2}\left(\ell_{1}^{2}+\ell_{2}^{2}\right) \Im^{\prime \prime}+\left(a_{2} \iota_{1}^{2}+b_{2} \iota_{2}^{2}\right) \Im^{3}=0 .
\end{aligned}
$$


Recall $\epsilon_{1}, \epsilon_{2} \in\{1,-1\}$. Again by (2.10)-(2.15), Case 1 and Case 2, we have:

$$
\iota_{1}=\epsilon_{1} \sqrt{\frac{2\left(b_{1} c_{2}-b_{2} c_{1}\right)\left(\ell_{1}^{2}+\ell_{2}^{2}\right)}{a_{1} b_{2}-a_{2} b_{1}}}, \quad \iota_{2}=\epsilon_{2} \sqrt{\frac{2\left(a_{2} c_{1}-a_{1} c_{2}\right)\left(\ell_{1}^{2}+\ell_{2}^{2}\right)}{a_{1} b_{2}-a_{2} b_{1}}}
$$

with $\left(\Im, k_{1}, k_{2}\right)$ as follows:

$$
\begin{aligned}
& \left(\tan \varpi, 2 c_{1}\left(\ell_{1}^{2}+\ell_{2}^{2}\right), 2 c_{2}\left(\ell_{1}^{2}+\ell_{2}^{2}\right)\right), \quad\left(\sec \varpi,-c_{1}\left(\ell_{1}^{2}+\ell_{2}^{2}\right),-c_{2}\left(\ell_{1}^{2}+\ell_{2}^{2}\right)\right), \\
& \left(\operatorname{coth} \varpi,-2 c_{1}\left(\ell_{1}^{2}+\ell_{2}^{2}\right),-2 c_{2}\left(\ell_{1}^{2}+\ell_{2}^{2}\right)\right), \quad\left(\operatorname{csch} \varpi, c_{1}\left(\ell_{1}^{2}+\ell_{2}^{2}\right), c_{2}\left(\ell_{1}^{2}+\ell_{2}^{2}\right)\right) ; \\
& \iota_{1}=m \epsilon_{1} \sqrt{\frac{2\left(b_{1} c_{2}-b_{2} c_{1}\right)\left(\ell_{1}^{2}+\ell_{2}^{2}\right)}{a_{1} b_{2}-a_{2} b_{1}}}, \quad \iota=m \epsilon_{2} \sqrt{\frac{2\left(a_{2} c_{1}-a_{1} c_{2}\right)\left(\ell_{1}^{2}+\ell_{2}^{2}\right)}{a_{1} b_{2}-a_{2} b_{1}}},
\end{aligned}
$$

$\Im=\operatorname{sn} \varpi$ and $\left(k_{1}, k_{2}\right)=-\left(1+m^{2}\right)\left(\ell_{1}^{2}+\ell_{2}^{2}\right)\left(c_{1}, c_{2}\right)$;

$$
\iota_{1}=m \epsilon_{1} \sqrt{\frac{2\left(b_{2} c_{1}-b_{1} c_{2}\right)\left(\ell_{1}^{2}+\ell_{2}^{2}\right)}{a_{1} b_{2}-a_{2} b_{1}}}, \quad \iota_{2}=m \epsilon_{2} \sqrt{\frac{2\left(a_{1} c_{2}-a_{2} c_{1}\right)\left(\ell_{1}^{2}+\ell_{2}^{2}\right)}{a_{1} b_{2}-a_{2} b_{1}}},
$$

$\Im=\mathrm{cn} \varpi$ and $\left(k_{1}, k_{2}\right)=\left(2 m^{2}-1\right)\left(\ell_{1}^{2}+\ell_{2}^{2}\right)\left(c_{1}, c_{2}\right)$;

$$
\iota_{1}=\epsilon_{1} \sqrt{\frac{2\left(b_{2} c_{1}-b_{1} c_{2}\right)\left(\ell_{1}^{2}+\ell_{2}^{2}\right)}{a_{1} b_{2}-a_{2} b_{1}}}, \quad \iota_{2}=\epsilon_{2} \sqrt{\frac{2\left(a_{1} c_{2}-a_{2} c_{1}\right)\left(\ell_{1}^{2}+\ell_{2}^{2}\right)}{a_{1} b_{2}-a_{2} b_{1}}},
$$

$\Im=\operatorname{dn} \varpi$ and $\left(k_{1}, k_{2}\right)=\left(2-m^{2}\right)\left(\ell_{1}^{2}+\ell_{2}^{2}\right)\left(c_{1}, c_{2}\right)$.

Theorem 3.7. Let $d_{1}, d_{2}, \ell_{1}, \ell_{2}, \ell_{3} \in \mathbb{R}$ and let $\epsilon_{1}, \epsilon_{2} \in\{1,-1\}$. We have the following solutions of the coupled two-dimensional cubic nonlinear Schrödinger equations (1.2) and (1.3):

$$
\begin{gathered}
\psi=\frac{\epsilon_{1} e^{\left(x^{2}+y^{2}\right) i / 4 c_{1} t}}{\ell_{1} x+\ell_{2} y+\ell_{3} t} \sqrt{\frac{2\left(b_{1} c_{2}-b_{2} c_{1}\right)\left(\ell_{1}^{2}+\ell_{2}^{2}\right)}{a_{1} b_{2}-a_{2} b_{1}}}, \\
\varphi=\frac{\epsilon_{2} e^{\left(x^{2}+y^{2}\right) i / 4 c_{2} t}}{\ell_{1} x+\ell_{2} y+\ell_{3} t} \sqrt{\frac{2\left(a_{2} c_{1}-a_{1} c_{2}\right)\left(\ell_{1}^{2}+\ell_{2}^{2}\right)}{a_{1} b_{2}-a_{2} b_{1}}} ; \\
\psi=\epsilon_{1} e^{\left(x^{2}+y^{2}\right) i / 4 c_{1} t} \sqrt{\frac{b_{1} c_{2}-b_{2} c_{1}}{\left(a_{1} b_{2}-a_{2} b_{1}\right)\left(\left(x-d_{1} t\right)^{2}+\left(y-d_{2} t\right)^{2}\right)}}, \\
\psi=\epsilon_{2} e^{\left(x^{2}+y^{2}\right) i / 4 c_{2} t} \sqrt{\frac{a_{2} c_{1}-a_{1} c_{2}}{\left(a_{1} b_{2}-a_{2} b_{1}\right)\left(\left(x-d_{1} t\right)^{2}+\left(y-d_{2} t\right)^{2}\right)}} \\
=\epsilon_{1} \sqrt{\frac{2\left(b_{1} c_{2}-b_{2} c_{1}\right)\left(\ell_{1}^{2}+\ell_{2}^{2}\right)}{a_{1} b_{2}-a_{2} b_{1}} \frac{1}{t} \tan \frac{\ell_{1} x+\ell_{2} y+\ell_{3} t}{t}} \\
\quad \times \exp \left(\frac{x^{2}+y^{2}}{4 c_{1} t}-\frac{2 c_{1}\left(\ell_{1}^{2}+\ell_{2}^{2}\right)}{t}\right) i
\end{gathered}
$$




$$
\begin{aligned}
\varphi= & \epsilon_{2} \sqrt{\frac{2\left(a_{2} c_{1}-a_{1} c_{2}\right)\left(\ell_{1}^{2}+\ell_{2}^{2}\right)}{a_{1} b_{2}-a_{2} b_{1}}} \frac{1}{t} \tan \frac{\ell_{1} x+\ell_{2} y+\ell_{3} t}{t} \\
& \times \exp \left(\frac{x^{2}+y^{2}}{4 c_{2} t}-\frac{2 c_{2}\left(\ell_{1}^{2}+\ell_{2}^{2}\right)}{t}\right) i
\end{aligned}
$$

$$
\begin{aligned}
\psi= & \epsilon_{1} \sqrt{\frac{2\left(b_{1} c_{2}-b_{2} c_{1}\right)\left(\ell_{1}^{2}+\ell_{2}^{2}\right)}{a_{1} b_{2}-a_{2} b_{1}}} \frac{1}{t} \sec \frac{\ell_{1} x+\ell_{2} y+\ell_{3} t}{t} \\
& \times \exp \left(\frac{x^{2}+y^{2}}{4 c_{1} t}+\frac{c_{1}\left(\ell_{1}^{2}+\ell_{2}^{2}\right)}{t}\right) i
\end{aligned}
$$

$$
\begin{aligned}
\varphi= & \epsilon_{2} \sqrt{\frac{2\left(a_{2} c_{1}-a_{1} c_{2}\right)\left(\ell_{1}^{2}+\ell_{2}^{2}\right)}{a_{1} b_{2}-a_{2} b_{1}}} \frac{1}{t} \sec \frac{\ell_{1} x+\ell_{2} y+\ell_{3} t}{t} \\
& \times \exp \left(\frac{x^{2}+y^{2}}{4 c_{2} t}+\frac{c_{2}\left(\ell_{1}^{2}+\ell_{2}^{2}\right)}{t}\right) i
\end{aligned}
$$

$$
\begin{aligned}
\psi= & \epsilon_{1} \sqrt{\frac{2\left(b_{1} c_{2}-b_{2} c_{1}\right)\left(\ell_{1}^{2}+\ell_{2}^{2}\right)}{a_{1} b_{2}-a_{2} b_{1}}} \frac{1}{t} \operatorname{coth} \frac{\ell_{1} x+\ell_{2} y+\ell_{3} t}{t} \\
& \times \exp \left(\frac{x^{2}+y^{2}}{4 c_{1} t}+\frac{2 c_{1}\left(\ell_{1}^{2}+\ell_{2}^{2}\right)}{t}\right) i
\end{aligned}
$$

$$
\begin{aligned}
\varphi= & \epsilon_{2} \sqrt{\frac{2\left(a_{2} c_{1}-a_{1} c_{2}\right)\left(\ell_{1}^{2}+\ell_{2}^{2}\right)}{a_{1} b_{2}-a_{2} b_{1}}} \frac{1}{t} \operatorname{coth} \frac{\ell_{1} x+\ell_{2} y+\ell_{3} t}{t} \\
& \times \exp \left(\frac{x^{2}+y^{2}}{4 c_{2} t}+\frac{2 c_{2}\left(\ell_{1}^{2}+\ell_{2}^{2}\right)}{t}\right) i
\end{aligned}
$$

$$
\begin{aligned}
\psi= & \epsilon_{1} \sqrt{\frac{2\left(b_{1} c_{2}-b_{2} c_{1}\right)\left(\ell_{1}^{2}+\ell_{2}^{2}\right)}{a_{1} b_{2}-a_{2} b_{1}}} \frac{1}{t} \operatorname{csch} \frac{\ell_{1} x+\ell_{2} y+\ell_{3} t}{t} \\
& \times \exp \left(\frac{x^{2}+y^{2}}{4 c_{1} t}-\frac{c_{1}\left(\ell_{1}^{2}+\ell_{2}^{2}\right)}{t}\right) i
\end{aligned}
$$

$$
\begin{aligned}
\varphi= & \epsilon_{2} \sqrt{\frac{2\left(a_{2} c_{1}-a_{1} c_{2}\right)\left(\ell_{1}^{2}+\ell_{2}^{2}\right)}{a_{1} b_{2}-a_{2} b_{1}}} \frac{1}{t} \operatorname{csch} \frac{\ell_{1} x+\ell_{2} y+\ell_{3} t}{t} \\
& \times \exp \left(\frac{x^{2}+y^{2}}{4 c_{2} t}-\frac{c_{2}\left(\ell_{1}^{2}+\ell_{2}^{2}\right)}{t}\right) i
\end{aligned}
$$

$$
\begin{aligned}
\psi= & m \epsilon_{1} \sqrt{\frac{2\left(b_{1} c_{2}-b_{2} c_{1}\right)\left(\ell_{1}^{2}+\ell_{2}^{2}\right)}{a_{1} b_{2}-a_{2} b_{1}}} \frac{1}{t} \operatorname{sn} \frac{\ell_{1} x+\ell_{2} y+\ell_{3} t}{t} \\
& \times \exp \left(\frac{x^{2}+y^{2}}{4 c_{1} t}+\frac{c_{1}\left(1+m^{2}\right)\left(\ell_{1}^{2}+\ell_{2}^{2}\right)}{t}\right) i
\end{aligned}
$$




$$
\begin{aligned}
& \varphi=m \epsilon_{2} \sqrt{\frac{2\left(a_{2} c_{1}-a_{1} c_{2}\right)\left(\ell_{1}^{2}+\ell_{2}^{2}\right)}{a_{1} b_{2}-a_{2} b_{1}}} \frac{1}{t} \operatorname{sn} \frac{\ell_{1} x+\ell_{2} y+\ell_{3} t}{t} \\
& \times \exp \left(\frac{x^{2}+y^{2}}{4 c_{2} t}+\frac{c_{2}\left(1+m^{2}\right)\left(\ell_{1}^{2}+\ell_{2}^{2}\right)}{t}\right) i \\
& \psi=m \epsilon_{1} \sqrt{\frac{2\left(b_{2} c_{1}-b_{1} c_{2}\right)\left(\ell_{1}^{2}+\ell_{2}^{2}\right)}{a_{1} b_{2}-a_{2} b_{1}}} \frac{1}{t} \operatorname{cn} \frac{\ell_{1} x+\ell_{2} y+\ell_{3} t}{t} \\
& \times \exp \left(\frac{x^{2}+y^{2}}{4 c_{1} t}-\frac{c_{1}\left(2 m^{2}-1\right)\left(\ell_{1}^{2}+\ell_{2}^{2}\right)}{t}\right) i \\
& \varphi=m \epsilon_{2} \sqrt{\frac{2\left(a_{1} c_{2}-a_{2} c_{1}\right)\left(\ell_{1}^{2}+\ell_{2}^{2}\right)}{a_{1} b_{2}-a_{2} b_{1}}} \frac{1}{t} \operatorname{cn} \frac{\ell_{1} x+\ell_{2} y+\ell_{3} t}{t} \\
& \times \exp \left(\frac{x^{2}+y^{2}}{4 c_{2} t}-\frac{c_{2}\left(2 m^{2}-1\right)\left(\ell_{1}^{2}+\ell_{2}^{2}\right)}{t}\right) i \text {; } \\
& \psi=\epsilon_{1} \sqrt{\frac{2\left(b_{2} c_{1}-b_{1} c_{2}\right)\left(\ell_{1}^{2}+\ell_{2}^{2}\right)}{a_{1} b_{2}-a_{2} b_{1}}} \frac{1}{t} \operatorname{dn} \frac{\ell_{1} x+\ell_{2} y+\ell_{3} t}{t} \\
& \times \exp \left(\frac{x^{2}+y^{2}}{4 c_{1} t}+\frac{c_{1}\left(m^{2}-2\right)\left(\ell_{1}^{2}+\ell_{2}^{2}\right)}{t}\right) i, \\
& \varphi=\epsilon_{2} \sqrt{\frac{2\left(a_{1} c_{2}-a_{2} c_{1}\right)\left(\ell_{1}^{2}+\ell_{2}^{2}\right)}{a_{1} b_{2}-a_{2} b_{1}}} \frac{1}{t} \operatorname{dn} \frac{\ell_{1} x+\ell_{2} y+\ell_{3} t}{t} \\
& \times \exp \left(\frac{x^{2}+y^{2}}{4 c_{2} t}+\frac{c_{2}\left(m^{2}-2\right)\left(\ell_{1}^{2}+\ell_{2}^{2}\right)}{t}\right) i \text {. }
\end{aligned}
$$

\section{References}

[AEK] N. Akhmediev, V. Eleonskii and N. Kulagin, First-order exact solutions of the nonlinear Schrödinger equation, Teoret. Mat. Fiz. 72 (1987), 183-196.

[GG] B. Grébert and J. Guillot, Periodic solutions of coupled nonlinear Schrödinger equations in nonlinear optics: the resonant case, Appl. Math. Lett. 9 (1996), 65-68.

[GW] L. Gagnon and P. Winternitz, Exact solutions of the cubic and quintic nonlinear Schrödinger equation for a cylindrical geometry, Phys. Rev. A 22 (1989), 296

[HS] F. Hioe and T. Salter, Special set and solutions of coupled nonlinear Schrödinger equations, J. Phys. A: Math. Gen. 35 (2002), no. 42, 8913-8928. 
[I] N. H. Ibragimov, Lie Group Analysis of Differential Equations, Volume 2, CRC Handbook, CRC Press, 1995.

[MP] D. Mihalache and N. Panoin, Exact solutions of nonlinear Schrödinger equation for positive group velocity dispersion, J. Math. Phys. 33 (1992), no. 6, 2323-2328.

[RL1] R. Radhakrishnan and M. Lakshmanan, Bright and dark soliton solutions to coupled nonlinear Schrödinger equations, J. Phys. A: Math. Gen. 28 (1995), no. 9, 2683-2692.

[RL2] R. Radhakrishnan and M. Lakshmanan, Exact soliton solutions to coupled nonlinear Schrödinger equations with higher-order effects, Phys. Rev. E(3) 54 (1995), no. 3, 2949-2955.

[SEG] E. Saied, R. EI-Rahman and M. Ghonamy, On the exact solution of $(2+1)-$ dimensional cubic nonlinear Schrödinger (NLS) equation", J. Phy. A: Math. Gen. 36 (2003), 6751-6770.

[WG] Z. Wang and D. Guo, Special functions, World Scientific, Singapore, 1998. 